Bull. Austral. Math. Soc.

VOL. 38 (1988) [339-344]

\title{
STABILITY OF LINEAR NEUTRAL DELAY-DIFFERENTIAL SYSTEMS
}

\section{LI-MING LI}

Sufficient conditions are obtained for the stability of linear neutral delay-differential systems by using a delay-differential inequality.

\section{INTRODUCTION}

This paper extends the result [2] for linear delay-differential systems to the neutral case:

$$
\dot{x}(t)=A x(t)+B x(t-\tau)+C \dot{x}(t-\tau) \quad(t \geq 0)
$$

where, $x(t)=\left(x,(t), x_{2}(t), \ldots, x_{n}(t)\right)^{T}, A, B$ and $C$ denote real constant $n \times n$ matrices with elements $a_{i j} . b_{i j} \cdot c_{i j}(i j=1,2, \ldots, n)$ respectively, and $\tau>0$ is a constant. We adopt the following norms for vectors $x=\left(x_{1}, x_{2}, \ldots, x_{n}\right)^{T}$ and matrices $A=$ $\left(a_{i j}\right)_{n \times n}$ respectively:

$$
\begin{aligned}
& \|x\|_{1}=\max _{i}\left|x_{i}\right|, \quad\|x\|_{2}=\left(\sum_{i=1}^{n} x_{i}^{2}\right)^{\frac{1}{2}}, \quad\|x\|_{\infty}=\sum_{i=1}^{n}\left|x_{i}\right|, \\
& \|A\|_{1}=\max _{j} \sum_{i=1}^{n}\left|a_{i} j\right|, \quad\|A\|_{2}=\left\{\lambda \max \left[A^{T} A\right]\right\}^{\frac{1}{2}}, \quad\|A\|_{\infty}=\max _{i} \sum_{j=1}^{n}\left|a_{i} j\right| .
\end{aligned}
$$

The measure $\mu(A)$ of a matrix $A$ is defined by

$$
\mu(A)=\lim _{h \rightarrow 0^{+}} \frac{\|I+h A\|-1}{h},
$$

which for the corresponding norms reduces to

$$
\begin{aligned}
\mu_{1}(A) & =\max _{j}\left[a_{i j}+\sum_{\substack{i=1 \\
i \neq j}}^{n}\left|a_{i j}\right|\right], \\
\mu(A) & =\frac{1}{2} \lambda_{\max }\left[A^{T}+A\right], \\
\mu_{\infty}(A) & =\max _{i}\left[a_{i i}+\sum_{\substack{j=1 \\
j \neq i}}^{n}\left|a_{i j}\right|\right],
\end{aligned}
$$

where $A^{T}$ denotes the transposed of $A, \lambda_{\max }[B]$ denotes the largest eignevalue of $B$, and $I$ denotes the unit matrix.

Received 13 January 1988

Copyright Clearance Centre, Inc. Serial-fee code: 0004-9729/88 \$A2.00+0.00. 


\section{A Delay-Differential INEQUality}

A delay-differential inequality was discussed in [1] and [4]. Here an extension is given

THEOREM 2.1. Let $p_{i}(t) \geqslant 0 \quad(i=1,2)$ be continuously differentiable functions on $[-\tau,+\infty)$ which satisfy

$$
\left\{\begin{aligned}
\dot{P}_{1}(t) & \leqslant a_{11} p_{1}(t)+a_{12} p_{2}(t)+b_{11} \widetilde{p_{1}(t)}+b_{12} \widetilde{p_{2}(t)} \\
0 & \leqslant a_{21} p_{1}(t)+a_{22} p_{2}(t)+b_{21} \widetilde{p_{1}(t)}+b_{22} \widetilde{p_{2}(t)}
\end{aligned}(t \geqslant 0)\right.
$$

where, $a_{i j} \geqslant 0(i \neq j), b_{i j} \geqslant 0, \widetilde{p_{i}(t)}=\sup _{t-r \leqslant \theta \leqslant t} p_{i}(\theta) \quad(i, j=1,2)$. If $a_{i i}+b_{i i}<0$ and the real parts of all eigenvalues of the matrix $\left(a_{i j}+b_{i j}\right)_{2 \times 2}$ are negative. Then there exist constants $M \geqslant 1, \alpha>0$ such that:

$$
p_{i}(t) \leqslant M\left(\sum_{j=1}^{2} \widetilde{p_{j}(0)}\right) e^{\alpha t} \quad t \in[-\tau, \infty)
$$

Proof: From the properties of a stable Metzler - Matrix [3], there exist constants $\alpha_{i}>0 \quad(i=1,2)$ such that:

$$
\begin{aligned}
& \left(a_{11}+b_{11}\right) \alpha_{1}+\left(a_{12}+b_{12}\right) \alpha_{2}<0 \\
& \left(a_{21}+b_{21}\right) \alpha_{1}+\left(a_{22}+b_{22}\right) \alpha_{2}<0 .
\end{aligned}
$$

We choose two constants, $\alpha>0$ and $k>0$, such that

$$
\begin{aligned}
\alpha \alpha_{1}+a_{11} \alpha_{1}+b_{11} \alpha_{1} e^{\alpha \tau}+a_{12} \alpha_{2}+b_{12} \alpha_{2} e^{\alpha \tau} & <0 \\
a_{21} \alpha_{1}+b_{21} \alpha_{1} e^{\alpha \tau}+a_{22} \alpha_{2}+b_{22} \alpha_{2} e^{\alpha \tau} & <0
\end{aligned}
$$

and

$$
k \alpha_{i} e^{-\alpha \tau}>1 \quad(i=1,2) .
$$

For a sufficiently small real numble $\varepsilon>0$, we define

$$
w_{i}(t)=k \alpha_{i}\left(\sum_{j=1}^{2} \widetilde{p_{j}(0)}+\varepsilon\right) e^{-\alpha t} \quad(i=1,2, t \geqslant-\tau) .
$$

It is easy to check that $p_{i}(t)<w_{i}(t) \quad(i=1,2, t \in[-\tau, 0])$. We want to prove that:

$$
p_{i}(t)<w_{i}(t) \quad(i=1,2, t \in[0,+\infty))
$$


If this inequality did not hold, then one of the following two situations would occur:

1. There exists a $t_{1}>0$, such that

$$
p_{1}(t)=w_{1}\left(t_{1}\right) \text { and } p_{i}(t) \leqslant w_{i}(t) \quad\left(i=1,2,-\tau \leqslant t \leqslant t_{1}\right)
$$

furthermore, $\dot{p}_{1}\left(t_{1}\right) \geqslant \dot{w}_{1}\left(t_{1}\right)$. Also, from (2.3), we have

$$
\begin{aligned}
\dot{p}_{1}\left(t_{1}\right) & \leqslant a_{11} w_{1}\left(t_{1}\right)+a_{12} w_{2}\left(t_{1}\right)+b_{11} \sup _{t_{1}-\tau \leqslant \theta \leqslant t_{1}} w_{1}(\theta)+b_{12} \sup _{t_{1}-\tau \leqslant \theta \leqslant t_{1}} w_{2}(\theta) \\
& =a_{11} w_{1}\left(t_{1}\right)+a_{12} w_{2}\left(t_{1}\right)+b_{11} w_{1}\left(t_{1}-\tau\right)+b_{12} w_{2}\left(t_{1}-\tau\right)
\end{aligned}
$$

so

$$
\begin{aligned}
\dot{w}_{1}\left(t_{1}\right) & =-k \alpha_{1} \alpha\left(\sum_{j=1}^{2} \widetilde{p_{j}(0)}+\varepsilon\right) e^{-\alpha t_{1}} \\
& >k\left(a_{11} \alpha_{1}+b_{11} \alpha_{1} e^{\alpha \tau}+a_{12} \alpha_{2}+b_{12} \alpha_{2} e^{\alpha \tau}\right)\left(\sum_{j=1}^{2} \widetilde{p_{j}(0)}+\varepsilon\right) e^{-\alpha t_{1}} \\
& =a_{11} w_{1}\left(t_{1}\right)+a_{12} w_{2}\left(t_{1}\right)+b_{11} w_{1}\left(t_{1}-\tau\right)+b_{12} w_{2}\left(t_{1}-\tau\right)
\end{aligned}
$$

That is, $\dot{w}_{1}\left(t_{1}\right)>\dot{p}_{1}\left(t_{1}\right)$. This contradicts $\dot{p}_{1}\left(t_{1}\right) \geqslant \dot{w}_{1}\left(t_{1}\right)$.

2. There exists a $t_{1}>0$, such that

$$
p_{2}\left(t_{1}\right)=w_{2}\left(t_{1}\right) \text { and } p_{i}(t) \leqslant w_{i}(t) \quad\left(i=1,2,-\tau \leqslant t \leqslant t_{1}\right) .
$$

Then from (2.3), we obtain

$$
\begin{aligned}
-a_{22} p_{2}\left(t_{1}\right) & \leqslant a_{21} w_{1}\left(t_{1}\right)+b_{21} w_{1}\left(t_{1}-\tau\right)+b_{22} w_{2}\left(t_{1}-\tau\right) \\
& =k\left(\sum_{j=1}^{2} \widetilde{p_{j}(0)}+\varepsilon\right) e^{-\alpha t_{1}}\left(a_{21} \alpha_{1}+b_{21} \alpha_{1} e^{\alpha \tau}+b_{22} \alpha_{2} e^{\alpha \tau}\right) \\
& <k\left(\sum_{j=1}^{2} p_{j}(0)+\varepsilon\right) e^{-\alpha t}\left(-a_{22} \alpha_{2}\right) \\
& =-a_{22} w_{2}\left(t_{1}\right)
\end{aligned}
$$

Since $-a_{22}>0$, we have $p_{2}\left(t_{1}\right)<w_{2}\left(t_{1}\right)$. This contradicts $p_{2}\left(t_{1}\right)=w_{2}\left(t_{1}\right)$.

Hence, we see that

$$
p_{i}(t)<w_{i}(t) \quad(i=1,2, t \geqslant 0) .
$$

Let $M=k\left(\alpha_{1}+\alpha_{2}\right)$ and $\varepsilon \rightarrow 0^{+}$. Then (2.2) is satisfied and the proof is complete.

Theorem 2.1 can be generalised immediately to the vector case: 
ThEOREM 2.2. Let $p_{i}(t) \geqslant 0 \quad(i=1,2, \ldots, 2 n)$ be continuously differentiable functions on $[-\tau,+\infty)$ such that $p^{*}(t)=\left(p_{1}(t), \ldots, p_{n}(t), 0 \ldots 0\right)^{T}$ and $p(t)=$ $\left(p_{1}(t), \ldots, p_{n}(t), p_{n+1}(t) \ldots p_{2 n}(t)\right)^{T}$ satisfies

$$
p^{*}(t) \leqslant A p(t)+\widetilde{B p(t)}
$$

where, $\widetilde{p(t)}=\left(\sup _{t-\tau \leqslant \theta \leqslant t} p_{1}(\theta), \ldots, \sup _{t-\tau \leqslant \theta \leqslant t} p_{2 n}(t)\right)^{T}$, and $A=\left(a_{i j}\right)_{2 n \times 2 n}, \quad B=$ $\left(b_{i j}\right)_{2 n \times 2 n}$, with $a_{i j} \geqslant 0(i \neq j) b_{i j} \geqslant 0 \quad(i, j=1,2, \ldots, 2 n)$. If $a_{i i}+b_{i i}<0$ and $\operatorname{Re\lambda }(A+B)<0$, then there exist constants $M \geqslant 1, \alpha>0$ such that:

$$
p_{i}(t) \leqslant M\left(\sum_{j=1}^{2 n} \sup _{-\tau \leqslant \theta \leqslant 0} p_{j}(\theta)\right) e^{-a t}, t \in[-\tau,+\infty) \quad i=1,2, \ldots, 2 n
$$

\section{A Stability Theorem}

By $C^{(1)}[-\tau, 0]$, we mean the Banach space of all functions $u(t)$ ( $u$ an $n$-vector) which are continuously differentiable on $[-\tau, 0]$ with norm:

$$
\|u\|_{\tau}=\sup _{-\tau \leqslant \theta \leqslant 0}\|u(\theta)\|+\sup _{-\tau \leqslant \theta \leqslant 0}\|\dot{u}(\theta)\|
$$

Consider the neutral delay-differential system

$$
\dot{x}(t)=A x(t)+B x(t-\tau)+C \dot{x}(t-\tau) .
$$

Let us define the stability of the solution $x=0$ for (3.1) as follows. Suppose that $\phi$ is a given continuously differentiable function on $[-\tau, 0]$ (that is $\phi \in C^{(1)}[-\tau, 0]$ ) and that $x(t)=x(t, \phi)$ on $[-\tau,+\infty)$ denotes the unique solution of (3.1) with $x(t)=$ $\phi(t), \dot{x}(t)=\dot{\phi}(t)$ for $t \in[-\tau, 0]$.

Definition 1: The solution $x=0$ of $(3.1)$ is stable in $C^{(1)}[-\tau, 0]$ if for each $\varepsilon>0$ there exists a $\delta=\delta(\varepsilon)>0$ such that $\|\phi\|_{\tau}<\delta$ implies that the solution $x(t, \phi)$ of (3.1) satisfies

$$
\|x(t, \phi)\|+\|\dot{x}(t, \phi)\|<\varepsilon \quad t \in[-\tau,+\infty)
$$

DEFINITION 2: The solution $x=0$ of $(3.1)$ is asymptotically stable in $C^{(1)}[-\tau, 0]$ if it is stable in $C^{(1)}\{-\tau, 0]$ and there exists $b_{0}>0$ such that $\|\phi\|_{\tau} \leqslant b_{0}$ implies

$$
\lim _{t \rightarrow+\infty}(\|x(t), \phi\|+\|\dot{x}(t, \phi)\|)=0
$$


Theorem 3.1. Suppose the coefficient matrices $A, B, C$ of (3.1) satisfy the following

$$
\|c\|<1 \text { and } \mu(A)+\frac{\|B\|+\|A\| \cdot\|C\|}{1-\|C\|}<0 .
$$

Then the solution $x=0$ of the system, (3.1) is asymptotically stable in $c^{(1)}[-\tau, 0]$ and there exist $M \geqslant 1, \alpha>0$ such that

$$
\|x(t, \phi)\|+\|\dot{x}(t, \phi)\| \leqslant 2 M\|\phi\|_{\tau} e^{-\alpha t}
$$

for every solution $x(t, \phi)$ of (3.1) with $x(t)=\phi(t), \dot{x}(t)=\dot{\phi}(t)$ on $t \in[-\tau, 0]$.

Proof: From the definition of the measure $\mu(A)$ we have for $t \in[0,+\infty)$,

$$
\begin{aligned}
\frac{d\|x(t)\|}{d t}-\mu(A)\|x(t)\| & =\lim _{h \rightarrow 0^{+}} \frac{1}{h}[\|x(t+h)\|-\|(I+h A)\| \cdot\|x(t)\|] \\
& \leqslant \lim _{h \rightarrow 0^{+}} \frac{1}{h}[\|x(t+h)-(I+h A) X(t)\|] \\
& \leqslant\|B\|\|x(t-\tau)\|+\|C\| \cdot\|\dot{x}(t-\tau)\|
\end{aligned}
$$

that is

$$
\frac{d\|x(t)\|}{d t} \leqslant \mu(A)\|x(t)\|+\|B\| \sup _{t-\tau \leqslant \theta \leqslant t}\|x(\theta)\|+\|C\| \sup _{t-\tau \leqslant \theta \leqslant t}\|\dot{x}(\theta)\| .
$$

From (3.1), we have directly

$$
0 \leqslant\|\dot{x}(t)\|+\|A\|\|x(t)\|+\|B\| \sup _{t-\tau \leqslant \theta \leqslant t}\|x(\theta)\|+\|C\| \sup _{t-\tau \leqslant \theta \leqslant t}\|\dot{x}(\theta)\| .
$$

Consider the functions $p_{1}(t), p_{2}(t)$ in Theorem 2.1 defined by

$$
p_{1}(t)=\|x(t)\|, p_{2}(t)=\|\dot{x}(t)\| \quad t \in[-\tau,+\infty) .
$$

It follows from (3.3) and (3.4) that

$$
\left\{\begin{aligned}
\dot{p}_{1}(t) & \leqslant \mu(A) p_{1}(t)+\|B\| \widetilde{p_{1}(t)}+\|C\| \widetilde{p_{2}(t)}, \\
0 & \leqslant\|A\| p_{1}(t)-p_{2}(t)+\|B\| \widetilde{p_{1}(t)}+\|C\| \widetilde{p_{2}(t)} .
\end{aligned}\right.
$$

We know from hypothesis (3.2) that the real parts of all eigenvalues of the matrix

$$
\left(\begin{array}{cc}
\mu(A)+\|B\| & \|C\| \\
\|A\|+\|B\| & -1+\|C\|
\end{array}\right)_{2 \times 2}
$$


are negative and $\mu(A)+\|B\|<0,-1+\|C\|<0$. Therefore, there exist $M \geqslant 1, \alpha>0$ such that

$$
p_{i}(t) \leqslant M\left(\sum_{j=1}^{2} \widetilde{p_{j}(0)}\right) e^{-\alpha t} \quad t \geqslant-\tau .
$$

By Theorem 2.1, we have

$$
\|x(t, \phi)\|+\|\dot{x}(t, \phi)\| \leqslant 2 M\|\phi\|_{\tau} e^{-\alpha t}, \quad t \geqslant-\tau
$$

and the proof is complete.

Consider the following system in component form

$$
\dot{x}_{i}(t)=a_{i i} x_{i}(t)+\sum_{\substack{j \neq i \\ j=1}}^{n} b_{i j} x_{j}(t-\tau)+\sum_{j=1}^{n} C_{i j} \dot{x}_{j}(t-\tau)
$$

where, $a_{i i}, b_{i j}(i \neq j), C_{i j} \quad(i=1,2, \ldots n)$ are constants and $a_{i i}<0 \quad(i=1,2, \ldots, n)$. Initating the proof of Theorem 3.1 by using the delay-differential inequality in vector form (Theorem 2.2 ), we can easily obtain the following theorem.

Theorem 3.2. Suppose that the coefficients of (3.5) satisfy the following

$$
a_{i i}+\sum_{\substack{j \neq i \\ j=1}}^{n}\left|b_{i j}\right|+\sum_{j=1}^{n}\left|C_{i j}\right|<0 \text { and }\left|a_{i j}\right|+\sum_{\substack{j \neq i \\ j=1}}^{n}\left|b_{i j}\right|+\sum_{j=1}^{n}\left|C_{i j}\right|<1
$$

$(i=1,2, \ldots, n)$. Then the solution $x_{i}=0$ of $(3.5)$ is asymptotically stable in $C^{(1)}[-\tau, 0]$.

Remark. Using Theorem 2.2, we can discuss the stability of the trivial solution for more complex neutral delay-differential systems.

\section{REFERENCES}

[1] R.D. Driver, 'Ordinary and Delay Differential Equations', in Appl. Math. Sci. 20 (Springer, New York, 1977).

[2] K. Gopalsamy, 'Some delays do not matter', Bull. Austral. Math. Soc. 30 (1984), 91-98.

[3] D.D. Siljak, Large-scale dynamic systems (North-Holland, New York, 1978).

[4] H. Tokumaru, N. Adachi and T. Amemiya, 'Macroscopic stability of interconnected systems', With discussions by Anthony N. Michael and G.S. Ladde and a reply by the author, in Proceedings of the IFAC 6th World Congress (Boston/Cambridge, Mass, 1975), Part1 Paper No. 44.4, 7, pp. A42-A43. in IFAc, Düsseldorf, 1975 (Authors' summary) 58. No.33213 93A15.

[5] M. Vidyasagar, Nonlinear systems analysis (Prentice Hall, Englewood Cliffs, New Jersy, 1978).

Teaching and Research Section of Mathematics

He Bei Institute of Finance and Economics

Shi Jia Zhuang

China 